This is a postprint version of the following published document:

de la Oliva, A., Hernández, J. A., Larrabeiti, D. \& Azcorra, A. (2016). An overview of the CPRI specification and its application to C-RANbased LTE scenarios. IEEE Communications Magazine, 54 (2), pp. 152-159.

DOI: $10.1109 / M C O M .2016 .7402275$

(c) 2016. IEEE. Personal use of this material is permitted. Permission from IEEE must be obtained for all other uses, in any current or future media, including reprinting/republishing this material for advertising or promotional purposes, creating new collective works, for resale or redistribution to servers or lists, or reuse of any copyrighted component of this work in other works. 


\title{
An Overview of the CPRI Specification and Its Application to C-RAN-Based LTE Scenarios
}

\author{
Antonio de la Oliva, José Alberto Hernández, David Larrabeiti, and Arturo Azcorra
}

\begin{abstract}
The CPRI specification has been introduced to enable the communication between radio equipment and radio equipment controllers, and is of particular interest for mobile operators willing to deploy their networks following the novel cloud radio access network approach. In such a case, CPRI provides an interface for the interconnection of remote radio heads with a baseband unit by means of the so-called fronthaul network. This article presents the CPRI specification, its concept, design, and interfaces, provides a use case for fronthaul dimensioning in a realistic LTE scenario, and proposes some interesting open research challenges in the next-generation $5 \mathrm{G}$ mobile network.
\end{abstract}

\section{INTRODUCTION AND MOTIVATION}

Mobile network operators (MNOs) have realized that the cloud radio access network (C-RAN) approach can provide a significant advantage with respect to their competitors in a market scenario where the trend in revenue per user is almost flat or decreasing. C-RAN has been recently introduced and further shown that significant operational expenditure (OPEX) and capital expenditure (CAPEX) reductions can be achieved with respect to traditional equipment deployments. A recent trial from China Mobile has shown 53 and 30 percent savings in OPEX and CAPEX, respectively [1].

The C-RAN approach advocates for the separation of the radio elements of the base station (called remote radio heads, RRHs) from the elements processing the baseband signal (called baseband units, BBUs), which are centralized in a single location or even virtualized into the cloud. This approach benefits from simpler radio equipment at the network edge, easier operation, and cheaper maintenance, while the main RAN intelligence (BBUs) is centralized in the operator-controlled premises. The challenge of C-RAN deployments is that such a functional split requires these two elements to be connected through a high-speed, low-latency, and accurately synchronized network, the so-called fronthaul. Such critical requirements are currently met with fiber optics $[2,3]$.

The C-RAN approach has some some clear benefits with respect to traditional integrated base stations (BSs). First, the cost of deploying
RRHs decreases considerably since the installation footprint is much smaller. RRHs do not need any refrigeration or costly on-site construction, thus shortening the time for deployment compared to traditional integrated BSs. On the other hand, BBUs can be aggregated and further virtualized in BBU pools. In this way, BBUs can be shared and turned off when necessary, reducing the cost of maintaining a network with low loads. Finally, another benefit of C-RAN is that it enables the use of cooperative radio techniques, cooperative multipoint (CoMP), allowing reduction of the interference between different radio transmissions and improving its performance. This further enables denser RRH deployments than traditional ones since interference among BSs can be better mitigated [4].

A number of radio equipment manufacturers have defined two main specifications for the transport of fronthaul traffic: the Common Public Radio Interface (CPRI) [5] and the Open Base Station Architecture Initiative (OBSAI). Both solutions are based on the implementation of the digital radio over fiber (D-RoF) concept, whereby the radio signal is sampled and quantized, and, after encoding, transmitted toward the BBU pool. These two specifications differ in the way that information is transmitted. CPRI is a serial line interface transmitting constant bit rate (CBR) data over a dedicated channel, while OBSAI uses a packet-based interface. The mapping methods of CPRI are more efficient than OBSAI [6], and most global vendors have chosen CPRI for their products.

The aim of this article is to present the CPRI specification, its concept, design, and interfaces, and further provide a guideline for fronthaul dimensioning in realistic Long Term Evolution (LTE) scenarios. We also provide some interesting open research challenges and current initiatives to bring the C-RAN concept to the fifth-generation $(5 \mathrm{G})$ mobile network. Accordingly, the following section briefly reviews the LTE physical layer (PHY) specifications required to understand the design of CPRI. We then introduce the top-level fronthaul network requirements demanded by CPRI and its main features, including the user plane data, control and management, and synchronization information multiplexing. After that we provide an application example of 
CPRI in a realistic LTE scenario. Finally, we conclude this work providing a number of open research issues and challenges regarding CPRI and the fronthaul.

\section{LTE PHYSICAL MEDIA}

This section presents the main features of the LTE PHY; in particular, LTE frequency-division duplex (LTE-FDD) is considered for brevity.

Concerning the downlink (DL), LTE uses orthogonal frequency-division multiple access (OFDMA), while in the uplink (UL) LTE uses single-carrier frequency-division multiple access (SC-FDMA). In both techniques data is encoded on multiple narrowband subcarriers, minimizing the negative effects of multi-path fading, distributing the interference effect across different users.

LTE allows spectrum flexibility where the channel bandwidth can be configured from 1.25 to $20 \mathrm{MHz}$. As an example, the DL with a 20 $\mathrm{MHz}$ channel and a $4 \times 4$ multiple-input multiple-output (MIMO) configuration can provide up to $300 \mathrm{Mb} / \mathrm{s}$ of user plane data. The UL peak data rate is $75 \mathrm{Mb} / \mathrm{s}$.

LTE defines a generic frame structure that applies to both DL and UL for FDD operation. Each LTE frame has a duration of $10 \mathrm{~ms}$, and is subdivided into 10 equal-size subframes of $1 \mathrm{~ms}$; each subframe comprises two slot periods of 0.5 ms duration. Depending on the cyclic prefix (CP) duration, each slot carries a number of orthogonal frequency-division multiplexing (OFDM) symbols ( 7 for the short $\mathrm{CP}$ or 6 for the long $\mathrm{CP}$ ) with $T_{\text {symbol }}=66.67 \mu \mathrm{s}$.

In the frequency domain, groups of $N_{s c}$ $=12$ adjacent subcarriers $(15 \mathrm{kHz} /$ subcarrier $)$ are grouped together on a slot-by-slot basis to form so-called physical resource blocks (PRBs), which are the smallest bandwidth unit $(180 \mathrm{kHz})$ assigned by the BS scheduler (Fig. 1). Thus, different transmission bandwidths use various PRBs per time slot, ranging from $N_{P R B}=6$ to 100 , as shown in Table 1.

Thus, each time slot carries a number of bits depending on the number of symbols per time slot (either 6 or 7), the modulation chosen, and the transmission bandwidth $B_{t x}$. For example, for $B_{t x}=2.5 \mathrm{MHz}$ (144 subcarriers) with 64-quadrature amplitude modulation (QAM) (6 b/symbol) and short $\mathrm{CP}\left(N_{C P}=7\right.$ OFDM symbols per time slot), the number of bits carried in a time slot of $0.5 \mathrm{~ms}$ duration is 6048 bits $(144$ subcarriers $\times 7$ OFDM symbols $\times 6 \mathrm{~b} / \mathrm{symbol}$ ), and the resulting data rate is approximately $12 \mathrm{Mb} / \mathrm{s}$. The effective data rate is actually less than this value since some resource elements of the PRB are reserved for control and signaling. It is also worth noting that there is one resource grid for each transmitting antenna; in other words, in a $2 \times 2$ MIMO configuration the value above doubles $(24 \mathrm{Mb} / \mathrm{s})$.

In order to recover all of the data transmitted, the receiver must take $N_{F F T}$ samples per OFDM symbol $\left(T_{\text {symbol }}\right)$ as specified in Table 1 . In the example above, the receiver must take $N_{F F T}$ $=256$ samples per OFDM symbol (66.67 s) in order to recover the data transmitted in $B_{t x}=2.5$ $\mathrm{MHz}$. In this case, the sampling frequency is $f_{s}=$ $3.84 \mathrm{MHz}\left(1.536 \cdot B_{t x}\right.$, as shown in the table), and the sampling period $T_{s}=1 / f_{s}=260.4141 \overline{6} \mathrm{~ns}$.

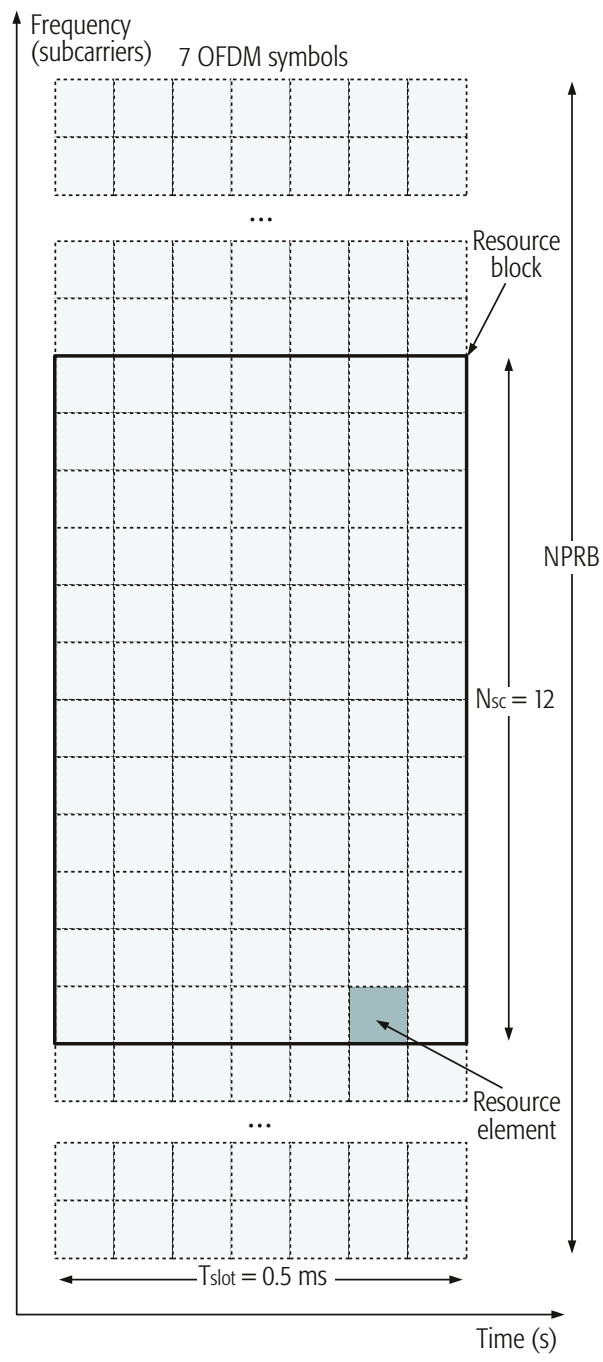

Figure 1. Downlink resource grid defined in LTE.

It is worth highlighting the importance of the $f_{s}=3.84 \mathrm{MHz}$ sampling reference value of LTE FDD, since the timing and synchronization design of CPRI revolves around this number. Essentially, $f_{c}=3.84 \mathrm{MHz}$ defines the main clock for CPRI framing, which is then oversampled to obtain the timing references for the other LTE channel bandwidths. ${ }^{1}$ In addition, one CPRI basic frame is generated every $1 / f_{c}=260.41 \overline{6} \mathrm{~ns}$ to carry the sampled digitized OFDM symbol, thus completely aligned with the LTE time reference.

\section{OVERVIEW OF CPRI \\ CONCEPT AND REQUIREMENTS}

According to the CPRI specification v6.1 [5], "the Common Public Radio Interface (CPRI) is an industry cooperation aimed at defining a publicly available specification for the key internal interface of radio base stations between the Radio Equipment Control (REC) and the Radio Equipment $(R E)$." In other words, the CPRI specification provides the physical (L1) and data link layer (L2) details for the transport of digitized radio information between REC and RE.

Figure 2 shows the functional split between REC and RE as defined in the CPRI specification
Concerning downlink,

LTE uses OFDMA, while in the uplink LTE uses SC-FDMA. In both techniques data is encoded on multiple narrowband subcarriers, minimizing the negative effects of multi-path fading, distributing the interference effect across different users.

1 The value of this clock is inherited from the single clock used in multimode WCDMA user equipments. 


\begin{tabular}{|c|c|c|c|c|c|c|}
\hline Tx BW (Btx) & $1.25 \mathrm{MHz}$ & $2.5 \mathrm{MHz}$ & $5 \mathrm{MHz}$ & $10 \mathrm{MHz}$ & $15 \mathrm{MHz}$ & $20 \mathrm{MHz}$ \\
\hline Number of PRB $\left(N_{P R B}\right)$ & 6 & 12 & 25 & 50 & 75 & 100 \\
\hline Sampling frequency & $1.92 \mathrm{MHz}$ & $3.84 \mathrm{MHz}$ & $7.68 \mathrm{MHz}$ & $15.36 \mathrm{MHz}$ & $23.04 \mathrm{MHz}$ & $30.72 \mathrm{MHz}$ \\
\hline Subcarriers/PRB $\left(N_{s c}\right)$ & \multicolumn{6}{|c|}{12} \\
\hline OFDM symbols $\left(N_{C P}\right)$ & \multicolumn{6}{|c|}{ 7/6 (Short/Long CP) } \\
\hline Modulation & \multicolumn{6}{|c|}{ QPSK, 16-QAM, 64-QAM } \\
\hline
\end{tabular}

Table 1. Downlink OFDM modulation parameters and CPRI bandwidth required for the case of $M=15 \mathrm{~b} / \mathrm{sample}$.

(DL). As shown in the figure, all the operations above the PHY and most of those of the PHY are performed by the REC, which generates the radio signal, samples it, and sends the resulting data to the RE. The RE basically reconstructs the waveform and transmits it over the air. The uplink case is similar, although the sampling of the radio signal must be performed in the RE. The main benefit of this split is that almost no digital processing functions are required at the RRHs, making them very small and cheap. In addition, the centralization of all the signal processing functions in the BBU simplifies the adoption of cooperative techniques such as CoMP, which require advanced processing of the radio signal of several RRHs simultaneously. Further discussion on alternative functional splits can be found in [7].

Some of the main design features and requirements of CPRI are listed below:

- CPRI supports a wide variety of radio standards: Third Generation Partnership Project (3GPP) Universal Terrestrial Radio Access (UTRA) FDD, WiMAX, 3GPP Evolved UTRA (E-UTRA, LTE), and 3GPP GSM/EDGE. This article only focuses on the use of CPRI for the transport of the E-UTRA interface.

- Although in most practical configurations CPRI will be configured in a point-to-point fashion, the specification also allows different topology configurations: star, chain, tree, ring, and multihop options to carry CPRI data over multiple hops. For example, CPRI natively supports the multiplexing of two CPRI-1 (614.4 Mb/s) into a single CPRI-2 (1228.8 Mb/s) frame through daisy chaining of the REs.

- CPRI requires strict synchronization and timing accuracy between REC and RE: the clock received at the $R E$ must be traceable to the main REC clock with an accuracy of $8.138 \mathrm{~ns}$. This number is exactly a fraction of $T_{c}=260.41 \overline{6}$, in particular $T_{c} / 32$.

- CPRI equipment must support an operating range of at least $10 \mathrm{~km}$.

- The main requirements for CPRI transmission apart from the required bandwidth are delay and bit error rate (BER). CPRI links should operate with at most $5 \mu$ s delay contribution excluding propagation delay, and a maximum allowed BER of $10^{-12}$. In addition, the frequency deviation from the CPRI link to the radio BS must be not larger than than $0.002 \mathrm{ppm}$.

\section{DESIGN AND IMPLEMENTATION}

CPRI defines three different logical connections between the REC and the RE: user plane data, control and management plane, and synchronization and timing. These three flows are multiplexed onto a digital serial communication line.

User Plane Data: Transported in the form of one or many in-phase and quadrature (IQ) data flows. Each IQ data flow reflects the radio signal, sampled and digitized, of one carrier at one independent antenna element, the so-called antenna carrier (AxC). In the particular case of LTE, an AxC contains one or more IQ samples for the duration of one UMTS chip $\left(T_{c}=1 / f_{c}=260.41 \overline{6}\right.$ ns since $f_{c}=3.84 \mathrm{MHz}$ ).

Synchronization Data Used for Time and Frame Alignment: The interface shall enable the RE to achieve the frequency accuracy specified in 3GPP TS 45.10 [8]. The central clock frequency generation in the RE shall be synchronized to the bit clock of one of the ports connecting $\mathrm{RE}$ and REC. With $8 \mathrm{~B} / 10 \mathrm{~B}$ or $64 \mathrm{~B} / 66 \mathrm{~B}$ line coding, the bit clock rate of the interface shall be a multiple of $38.4 \mathrm{MHz}$ in order to allow for a simple synchronization mechanism and frequency regeneration.

Control and Management: $C \& M$ data can be transmitted by either an in-band protocol (for time-critical signaling data) or higher-layer protocols not defined by CPRI. The inband protocol is used for synchronization and timing, and also for error detection/correction. This makes use of the line codings specified in IEEE 802.3 (line codes $8 \mathrm{~B} / 10 \mathrm{~B}$ and $64 \mathrm{~B} / 66 \mathrm{~B}$ ). The physical layer is capable of detecting link failures and synchronization issues as a result of line code violations.

Vendor-Specific: CPRI reserves some time slots for the transmission of any vendor-specific data, allowing manufacturers to customize their solutions. 


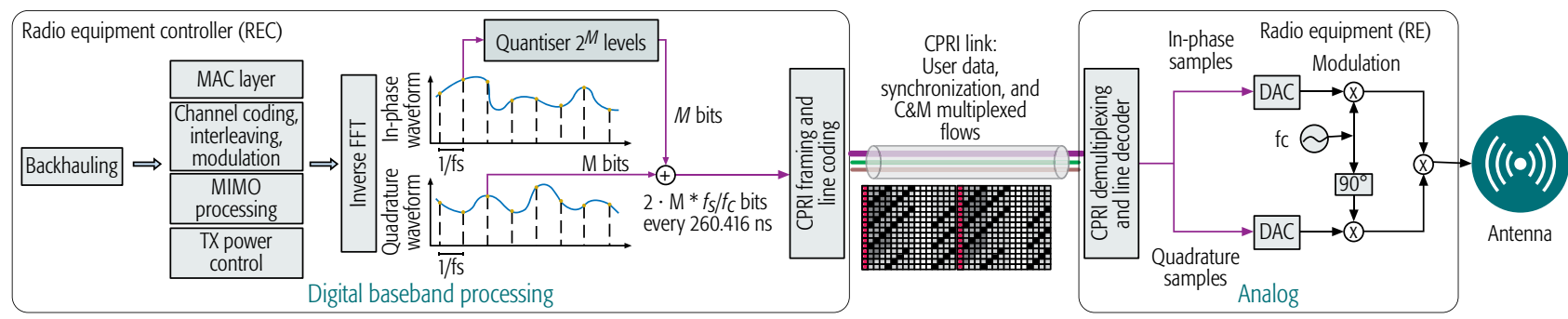

Figure 2. Conceptual explanation of REC/RE functional split.

\section{TRANSMISSION OF USER PLANE DATA}

The transmission of user plane data is based on the concept of an antenna carrier (AxC). Given that the LTE radio signal is first sampled and then quantized (Fig. 2), the amount of information carried by an $\mathrm{AxC}$ depends on two parameters:

- The sampling frequency $f_{s}$, which is a multiple of the nominal chip rate $f_{c}=3.84 \mathrm{MHz}$ (Table 1).

- The number of bits $M$ used in the quantization process of the $\mathrm{I}$ and $\mathrm{Q}$ radio signals. In E-UTRA, $M=8, \ldots, 20$ either DL or UL. Previous work [9] and actual field programmable gate array (FPGA) implementation of CPRI consider $M=15$ for capacity efficiency.

For example, in a configuration with $M=15$ $\mathrm{b} /$ sample, one AxC comprises $15+15=30 \mathrm{~b} / \mathrm{IQ}$ sample, which are transmitted in the following interleaved sequence:

$$
I_{0} Q_{0} I_{1} Q_{1} \ldots I_{M-1} Q_{M-1},
$$

that is, from the least significant bit (LSB) to the most significant bit (MSB).

In CPRI, one basic frame is created and transmitted every $T_{c}=260.41 \overline{6} \mathrm{~ns}$, which is based on the Universal Mobile Telecommunications System (UMTS) clock rate, that is, $3.84 \mathrm{MHz}$. This duration remains constant for all CPRI line bit rate options. As already indicated, this value of $T_{c}$ is designed to transport one fast Fourier transform (FFT) sample for an LTE channel bandwidth of $2.5 \mathrm{MHz}$, two samples for the 5 $\mathrm{MHz}$ bandwidth, four samples for the $10 \mathrm{MHz}$ channel, and so on.

A basic frame comprises $W=16$ words $(w$ $=0, \ldots, 15)$ whereby the length $T$ of each word depends on the CPRI line bit rate option (Table 2 ). The exact line bit rate values for each option are computed in the second column of Table 2 . In all cases, the first word $w=0$ is reserved for control, while the other 15 words are used to carry IQ data samples. For example, in CPRI option 1 , there is room for 120 (= 15 words $\times$ $8 \mathrm{~b}$ /word) bits for transporting the IQ samples of several AxCs. Thus, in a configuration of $2 M$ $=30 \mathrm{~b} / \mathrm{AxC}$, one basic frame can carry up to 4 AxCs consisting of one sample each. This is a basic configuration for an antenna serving four sectors with $2.5 \mathrm{MHz}$ LTE channel bandwidth. It is worth remarking that four $2.5 \mathrm{MHz}$ AxCs carry about $4 \cdot 12=48 \mathrm{Mb} / \mathrm{s}$ of actual LTE data, and are spread over $614.4 \mathrm{Mb} / \mathrm{s}$ after CPRI encapsulation; this is about 13 times higher bit rate.
CPRI defines a hierarchical framing with three layers (Fig. 3), chosen this way to match the framing numbers of the LTE FDD frame structure:

- Basic frame, of variable size, created and transmitted every $T_{c}=260.416 \mathrm{~ns}$.

- Hyperframe, which is a collection of 256 basic frames. One hyperframe is created every $256 \times T_{c}=66.67 \mu \mathrm{s}$, which is the OFDM symbol time in LTE. Thus, a hyperframe carries all the FFT samples required to decode the whole OFDM symbol.

- CPRI frame, which is a collection of 150 hyperframes. A CPRI frame is created every $10 \mathrm{~ms}$ and carries the digital samples of a whole LTE frame.

\section{CONTROL AND MANAGEMENT, AND SYNCHRONIZATION}

As noted before, the first word $(w=0)$ in every basic frame (control word) carries C\&M information; thus, 256 control words are available per hyperframe. These 256 control words are organized into 64 subchannels of 4 control words each (Fig. 4). As shown, every control word can be addressed by a subchannel ID $(0, \ldots, 63)$.

Each subchannel belongs to one category out of seven:

Synchronization: The control word on the first basic frame (CW 0 in Fig. 4) is reserved to indicate the starting of a new hyperframe. This control word uses a special $8 \mathrm{~B} / 10 \mathrm{~B}$ (K28.5) or $64 \mathrm{~B} / 66 \mathrm{~B}(50 \mathrm{~h})$ code. The three remaining words in the synchronization subchannel (words 64 , 128 , and 192) are used to signal the hyperframe number and the node $\mathrm{B}$ frame number (BFN) for synchronization purposes with the LTE framing.

L1 In-Band Protocol: Subchannel 2 carries the necessary signaling required to set up the different C\&M links, including starting up, resetting, and tearing down the CPRI link, and also to handle alarms at the PHY for different events such as loss of synchronization.

Slow C\&M Link: The subchannels assigned to this category enable the transmission of high-level data link control (HDLC) frames. HDLC is a well-known layer 2 protocol providing basic functionalities such as flow control and error correction based on retransmission.

Ctrl_AxC: A Ctrl_AxC designates one AxC-specific control data stream. The mapping of Ctrl_AxCs to AxCs as well as the actual content of the control data bytes are not defined in CPRI but are vendor-specific.

Fast C\&M Link: In addition to the slow C\&M link, the operator of the CPRI link is provided 
Number of AxCs of channel bandwidth and bit rate required per $\mathrm{AxC}$

\begin{tabular}{|c|c|c|c|c|c|c|c|c|c|}
\hline Option \# & $\begin{array}{l}\text { CPRI data } \\
\text { rate }(\mathrm{Mb} / \mathrm{s})\end{array}$ & Coding & $T$ & $\begin{array}{c}1.25 \mathrm{MHz} \\
(76.8 \mathrm{Mb} / \mathrm{s})\end{array}$ & $\begin{array}{c}2.5 \mathrm{MHz}(153.6 \\
\mathrm{Mb} / \mathrm{s})\end{array}$ & $\begin{array}{c}5 \mathrm{MHz}(307.2 \\
\mathrm{Mb} / \mathrm{s})\end{array}$ & $\begin{array}{c}10 \mathrm{MHz}(614.4 \\
\mathrm{Mb} / \mathrm{s})\end{array}$ & $\begin{array}{c}15 \mathrm{MHz}(921.6 \\
\mathrm{Mb} / \mathrm{s})\end{array}$ & $\begin{array}{c}20 \mathrm{MHz}(1228.8 \\
\mathrm{Mb} / \mathrm{s})\end{array}$ \\
\hline 1 & 614.4 & $8 \mathrm{~B} / 10 \mathrm{~B}$ & 8 & 8 & 4 & 2 & 1 & - & - \\
\hline 2 & 1228.8 & $8 \mathrm{~B} / 10 \mathrm{~B}$ & 16 & 16 & 8 & 4 & 2 & 1 & 1 \\
\hline 3 & 2457.6 & $8 \mathrm{~B} / 10 \mathrm{~B}$ & 32 & 32 & 16 & 8 & 4 & 2 & 1 \\
\hline 5 & 4915.2 & $8 \mathrm{~B} / 10 \mathrm{~B}$ & 64 & 64 & 32 & 16 & 8 & 5 & 4 \\
\hline 6 & 6144 & $8 \mathrm{~B} / 10 \mathrm{~B}$ & 80 & 80 & 40 & 20 & 10 & 6 & 5 \\
\hline 7 & 9830.4 & $8 \mathrm{~B} / 10 \mathrm{~B}$ & 128 & 128 & 64 & 32 & 16 & 10 & 8 \\
\hline 9 & 12165.12 & $64 \mathrm{~B} / 66 \mathrm{~B}$ & 192 & 192 & 96 & 48 & 24 & 16 & 12 \\
\hline
\end{tabular}

Table 2. Maximum number of AxC transported in a CPRI link, $M=15$ bits.

with a fast $C \& M$ subchannel to transmit other control information. Such control frames are first encapsulated over Ethernet and then transmitted over this subchannel. Fragmentation and reassembly are needed. For this purpose, CW 194 carries a pointer to the $\mathrm{CW}$ in the hyperframe containing the first byte of the Ethernet frame (shown in Fig. 4 as pointer P).

Reserved for Future Use and Vendor-Specific.

\section{CPRI FRONTHAUL DIMENSIONING IN C-RAN SCENARIOS}

\section{GeNeRAL DimENSIONING GUIDELINES}

Following the discussion earlier, the D-RoF transmission (i.e., sampling and quantization) of an $\mathrm{AxC}$ requires a data bit rate of $\mathrm{B}_{\mathrm{AxC}}=(2 M)$ $f_{s} \mathrm{~b} / \mathrm{s}$, expanded by factors $16 / 15$ (15 words data, 1 word C\&M) and either $10 / 8$ or $66 / 64(8 \mathrm{~B} / 10 \mathrm{~B}$ or $66 \mathrm{~B} / 64 \mathrm{~B}$ line coding, respectively). According to this, a $2.5 \mathrm{MHz}$ LTE channel requires 153.6 $\mathrm{Mb} / \mathrm{s}$ per AxC.

In this light, Table 2 shows the bit rate required per $\mathrm{AxC}$ for different LTE bandwidths and the maximum number of AxCs transported for standard CPRI bit rates. This table provides a good starting point for dimensioning fronthaul networks in C-RAN scenarios, and should be read as follows: CPRI option $6(6144 \mathrm{Mb} / \mathrm{s})$ can carry 80 AxCs@1.25 MHz LTE bandwidth, 40 AxC@2.5 MHz, or 5 AxC@20 MHz. On the other hand, if the LTE setup is fixed to a number of 3 sectors and $2 \times 2$ MIMO @ $10 \mathrm{MHz}$ LTE bandwidth (i.e., $2 \times 3$ AxCs), a lookup in Table 2, column " $10 \mathrm{MHz}$ LTE bandwidth" reveals that at least CPRI option 5 is required to carry such a number of $\mathrm{AxC}$.

\section{USE CASE: CPRI DOWNLINK REQUIREMENTS FOR A FOUR-}

AntenNa Site, $2 \times 2$ MIMO, $20 \mathrm{MHz}$ ChanNel SCenario

Consider the four-antenna/four-sector scenario operating an LTE $2 \times 2$ MIMO channel of 20 $\mathrm{MHz}$ bandwidth depicted in Fig. 3a. This sce- nario requires the multiplexing and transmission of four AxC groups (one per sector), while each AxC group comprises two AxCs, as shown in the figure.

Figure $3 b$ shows the amount of information carried in each AxC. As shown, one IQ sample $(2 \mathrm{M}=30 \mathrm{bits})$ is generated every $1 / f_{s}$, where $f_{s}$ $=30.72 \mathrm{MHz}$ for $20 \mathrm{MHz}$ LTE channels (Table 1 ). Thus, a total of $8 \times 30=240$ bits are generated every $1 / f_{s}$. It is also worth remarking that $f_{s}$ $=30.72 \mathrm{MHz}$ is exactly $8 f_{c}$; hence, $8 \mathrm{IQ}$ samples are generated every $1 / f_{c}=T_{c}=260.416 \mathrm{~ns}$ (i.e., $1920 \mathrm{IQ} \mathrm{b} / T_{c}$ total). This amount of information requires $8 \times 1228.8 \mathrm{Mb} / \mathrm{s}=9830.4 \mathrm{Mb} / \mathrm{s}(8 \mathrm{~B} / 10 \mathrm{~B}$ assumed), which is CPRI option 7 in Table 2. Alternatively, CPRI option 7A is also suitable for carrying the same 8 AxCs@20 MHz LTE channel and even requires slightly less bandwidth since $64 \mathrm{~B} / 66 \mathrm{~B}$ is used. In both cases, a $10 \mathrm{~Gb} / \mathrm{s}$ Ethernet transceiver is suitable as a physical medium for this scenario. ${ }^{2}$

Figure $3 c$ shows how the different $\mathrm{AxC}$ are grouped together and multiplexed over the line. The CPRI specification defines three mapping methods to multiplex different AxCs; we have chosen mapping method 3, which is backward compatible with previous CPRI specifications. Essentially, the IQ samples are arranged in order per $\mathrm{AxC}$ group (group 1 first, group 4 last) and interleaved within the group ( 30 bits $\mathrm{AxC} 0$, then 30 bits $\mathrm{AxC} 1$, then 30 bits $\mathrm{AxC} 0$ again, etc. for group 1).

Such ordering is then used to construct a CPRI basic frame (Fig. 3d) noting that one word for C\&M is added ahead of the 1920 data bits. One basic frame is constructed this way every $260.41 \overline{6} \mathrm{~ns} ; 256$ basic frames form a hyperframe $(66.67 \mu \mathrm{s})$, which includes the information of one LTE OFDM symbol; and 150 hyperframes form a super frame, which is synchronized with the 10 ms LTE frame (Fig. 3e).

Other scenarios would follow the same guidelines as before. For instance, the same configu- 


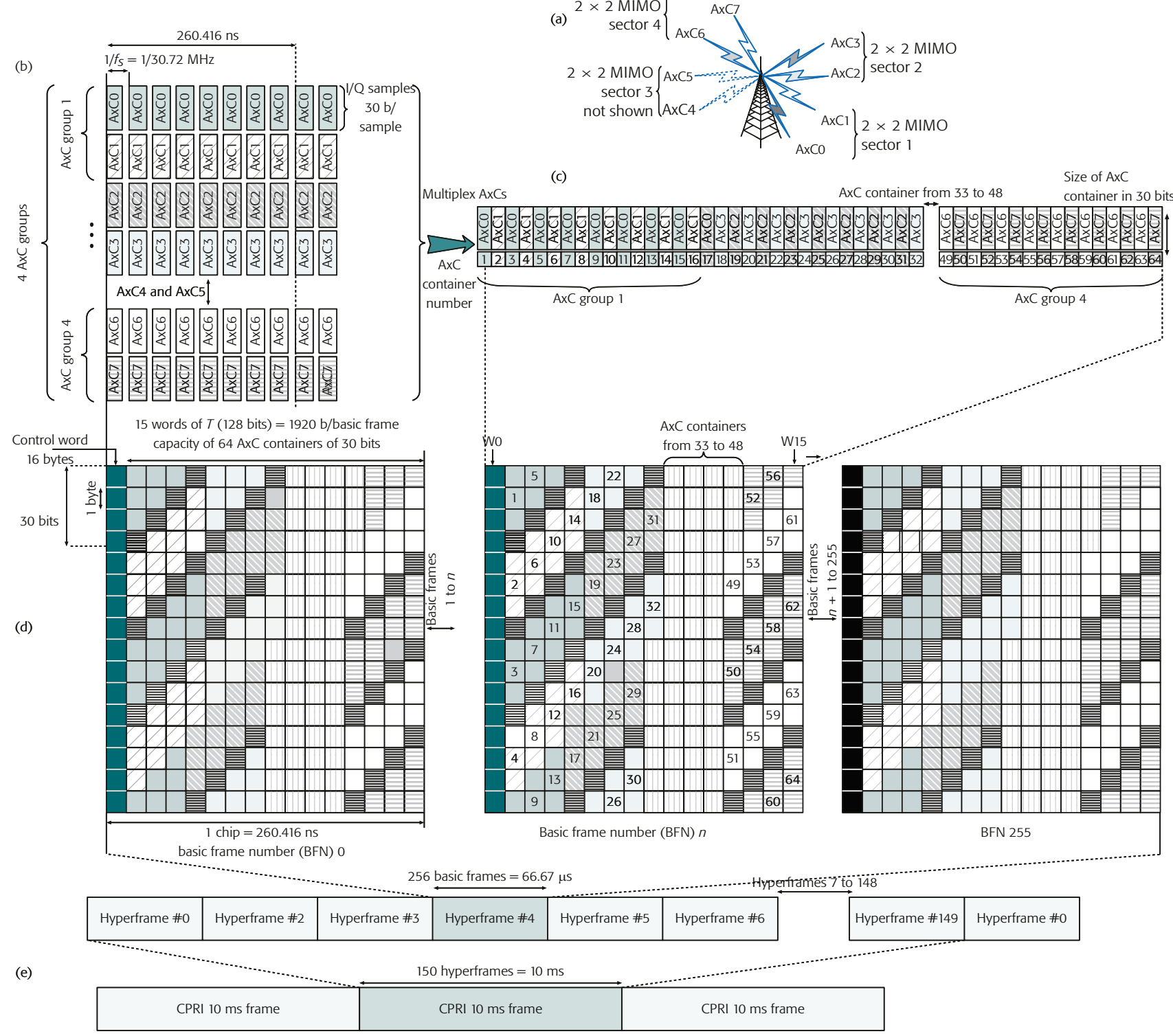

Figure 3. CPRI multiplexing of AxC data in a $2 \times 2$ MIMO $20 \mathrm{MHz}$ channel use case: a) scenario; b) AxC generation; c) AxC arrangement and serialization; d) basic frame construction; e) hyperframes and $10 \mathrm{~ms}$ CPRI frame.

ration in a $4 \times 4$ MIMO scenario would require the same sampling frequency $f_{s}$, but the data rate would double since we now have $4 \mathrm{AxC}$ groups with 4 AxCs per group, that is, a total of 16 AxCs. The arrangement of Fig. $3 \mathrm{c}$ would be the same for the AxC group (group 1 first, group 4 last), but $\mathrm{AxCs}$ within the group would alternate $(\mathrm{AxC} 0, \mathrm{AxC} 1, \mathrm{AxC} 2, \mathrm{AxC} 3, \mathrm{AxC} 0$ again, and so on for group 1).

\section{SUMMARY, CHALLENGES AND FUTURE RESEARCH}

This work has provided a short overview of CPRI, including concept, design, specification, and use case in an LTE C-RAN-based environment. The concept of C-RAN has recently appeared in the market, and the idea of separating RECs (BBUs) from REs (RRHs) is gaining traction in the mobile network industry.

On the research side, there is a common con- sensus on the key challenges of CPRI technology [10]. First, the amount of bandwidth required to transmit the radio signal is simply overwhelming for LTE. Moreover, the upcoming 5G RANs, where $100 \mathrm{MHz}$ channels with massive MIMO are envisioned, may require several tens or even hundreds of gigabits per second capacity in the fronthaul [11]. As an example, an $8 \times 8$ MIMO antenna covering four sectors produces $32 \mathrm{AxCs}$, which translate into around $32 \mathrm{~Gb} / \mathrm{s}$ for $20 \mathrm{MHz}$ bandwidth channels. In the case of $100 \mathrm{MHz}$ LTE channels, this same scenario requires five times (i.e., $160 \mathrm{~Gb} / \mathrm{s}$ ) the previous CPRI bandwidth.

Second, CPRI is a serial CBR interface with new frames transmitted every $T_{c}=260.41 \overline{6} \mathrm{~ns}$. This, together with the low-latency and strict synchronization requirements demanded, makes it very challenging to have CPRI and other traffic sources over the same link. Recent studies have approached this problem focusing on band- 


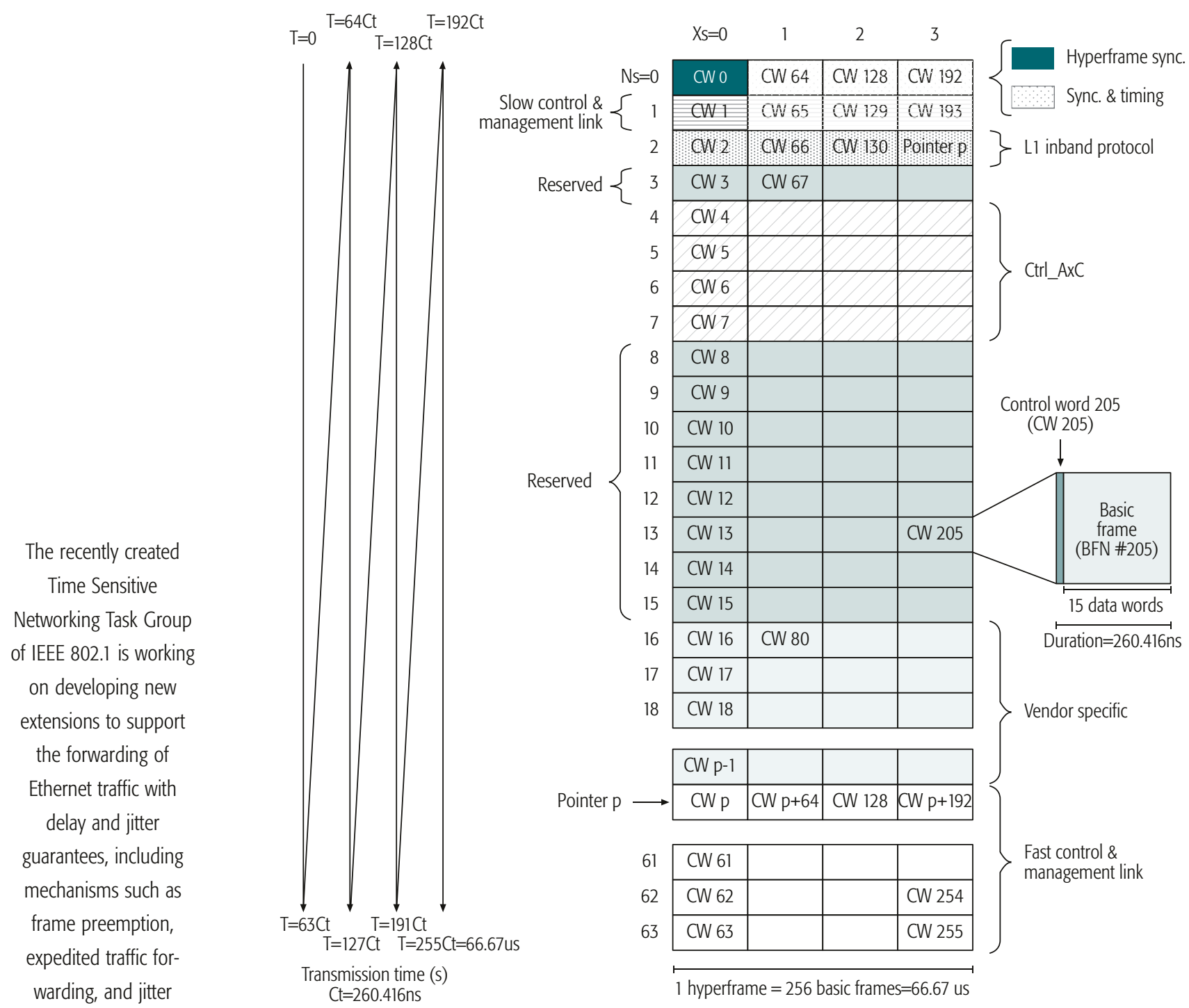

reduction techniques,

mainly buffering.

Figure 4. CPRI multiplexing of C\&M channels in the hyperframe. C\&M information is carried in the control word $(\mathrm{CW})$ of each CPRI frame.

width compression techniques. For example, the authors in [12] claim to provide about $1 / 5$ compression ratios within the $5 \mu$ s delay budget allowed by CPRI, thus significantly reducing the link load.

Bandwidth compression is indeed a starting point toward the packetization of CPRI data, via Ethernet framing, for instance. However plain Ethernet is asynchronous and best effort, and therefore not suitable as such for the transport of CPRI traffic. In this light, the recently created Time Sensitive Networking (TSN) Task Group of IEEE $802.1^{3}$ is working on developing new extensions to support the forwarding of Ethernet traffic with delay and jitter guarantees, including mechanisms such as frame preemption, expedited traffic forwarding, and jitter reduction techniques, mainly buffering [13].

In addition, the use of synchronous Ethernet seems mandatory in multihop scenarios [14]. Nevertheless, although high-precision timing protocols over Ethernet exist (see IEEE 1588v2), their accuracy is in the range of a few hundred nanoseconds, while CPRI requires at most tens of nanoseconds between REC and RE. New approaches using frequency adjustable oscillators or GPS signals are under study to solve this issue.

Finally, both research projects and standardization bodies (e.g., IEEE 1904.3 Standard for Radio over Ethernet Encapsulation and Mappings $^{4}$ ) are exploring the possible gains of redefining the RE/REC functional split of C-RAN in the next-generation networks [15]. Examples include the decoupling of fronthaul bandwidth and antenna number by moving antenna related operations to the RE (DL antenna mapping, FFT, etc.), or enabling traffic-dependent bandwidth adaptation by effectively coupling fronthaul bandwidth with the actual traffic served in the cell. The latter relies on the fact that many cell processing functions do not depend on the number of users, including FFT, cyclic prefix addition/removal, synchronization signals, and so on. More information about this novel approach can be found in [7]. 


\section{ACKNOWLEDGMENTS}

The authors would like to acknowledge the support of projects CRAMnet (grant no. TEC201238362-C03-01) and EU H2020 5G-Crosshaul Project (grant no. 671598) to the development of this work.

\section{REFERENCES}

[1] "C-RAN: The Road towards Green RAN," China Mobile White Paper, v2, 2011

[2] A. Lometti et al., "Backhauling Solutions for LTE Networks," Proc. 16th Int' Conf. Transparent Optical Networks, 2014, July 2014, pp. 1-6.

[3] A. Pizzinat et al., "Things You Should Know About Fronthaul," IEEE/OSA J. Lightwave Tech., 2015.

[4] R. Irmer et al., "Coordinated Multipoint: Concepts, Performance, and Field Trial Results," IEEE Commun. Mag., vol. 49, no. 2, Feb. 2011, pp. 102-11.

[5] Specification, CPRI, "V6.1 Common Public Radio Interface (CPRI); Interface Specification," NEC Corp., Nortel Networks SA, Siemens Networks GmbH \& Co. KG, Ericsson AB, and Huawei Technologies Co Ltd., July, 2014, 129 pages.

[6] M. Nahas et al., "Base Stations Evolution: Toward 4G Technology," Proc. IEEE Int'l. Conf. Telecommun., 2012, pp. 1-6.

[7] D. Wubben et al., "Benefits and Impact of Cloud Computing on 5G Signal Processing: Flexible Centralization through Cloud-RAN," IEEE Signal Processing Mag., vol. 31, no. 6, Oct. 2014, pp. 35-44.

[8] 3GPP, "3GPP TS 45.010: Radio Subsystem Synchronization," Rel. 10, V10.1.0, 2011.

[9] C. F. A. Lanzani, L. Dittmann, and M. S. Berger, 4G Mobile Networks: An Analysis of Spectrum Allocation, Software Radio Architectures and Interfacing Technology, Ph.D. dissertation, Tech. Univ. Denmark, Dept of Photonics Engineering.

[10] A. Saadani et al., "Digital Radio over Fiber for LTE-Advanced: Opportunities and Challenges," Proc. 17th IEEE Int'l. Conf. Optical Network Design and Modeling, 2013, 2013, pp. 194-99.

[11] J. E. Mitchell, "Integrated Wireless Backhaul over Optical Access Networks," IEEE/OSA J. Lightwave Technology, vol. 32, no. 20, Oct. 2014 pp. 3373-82.

[12] B. Guo et al., "CPRI Compression Transport for LTE and LTE-A Signal in C-RAN," Proc. CHINACOM, vol. 0, 2012, pp. 843-49.

[13] T. Wan and P. Ashwood, "A Performance Study of CPRI over Ethernet;" http://www.ieee1904.org/3/meetingarchive/2015/02/tf31502ashwoodla. pdf

[14] J. Aweya, "Implementing Synchronous Ethernet in Telecommunication Systems," IEEE Commun. Surveys \& Tutorials, vol. 16, no. 2, 2nd qtr. 2014, pp. 1080-1113.

[15] P. Rost et al., "Cloud Technologies for Flexible 5G Radio Access Networks," IEEE Commun. Mag., vol. 52, no. 5, May 2014, pp. 68-76.

\section{BIOGRAPHIES}

ANTONIO DE LA OLIVA (aoliva@it.uc3m.es) received his telecommunications engineering degree in 2004 and his PhD. in 2008 from Universidad Carlos III Madrid (UC3M), Spain, where he has been working as an associate professo since. His current line of research is related to networking in extremely dense networks. He is an active contributor to IEEE 802, where he has served as Vice-Chair of IEEE 802.21b and Technical Editor of IEEE 802.21d. He has also served as a Guest Editor of IEEE Communications Magazine.

José AlBERTO HernÁNDEZ (jahgutie@it.uc3m.es) completed his five-yea degree in telecommunications engineering at UC3M in 2002, and his Ph.D. degree in computer science at Loughborough University,Leicester, United Kingdom, in 2005. He has been a senior lecturer in the Department of Telematics Engineering since 2010, where he combines teaching and research in the areas of optical WDM networks, next-generation access networks, metro Ethernet, energy efficiency, and Hybrid optical-wireless technologies. He has published more than 75 articles in both journals and conference proceedings on these topics. He is a co-author of the book Probabilistic Modes for Computer Networks: Tools and Solved Problems.

DAVID LARRABEITI is a professor of switching and networking architectures at UC3M since 1998. He has participated in EU-funded research projects related to next-generation networks and protocols since FP6. He was UC3M Principal Investigator of the FP7 BONE European network of excellence on optical networking. He is currently involved in the FP7 Fed4FIRE and H2020 5G-Crosshaul research projects, participating in the development of technology and testbeds for backhaul network design.

ARTURO AzCORRA received his M.Sc. degree in telecommunications engineering from Universidad Politécnica de Madrid (UPM) in 1986 and his Ph.D. from the same university in 1989. In 1993, he obtained an M.B.A. with honors from Instituto de Empresa He has participated in and directed 49 research and technological development projects, including European ESPRIT, RACE, ACTS, and IST programs. He coordinated the CONTENT and E-NEXT European Networks of Excellence, and the CARMEN EU project. In addition to his scientific achievements, he has a relevant track record of research management. He was deputy vice rector of Academic Infrastructures at UC3M from 2000 to 2007. He served as direc tor general for Technology Transfer and Corporate Development at the Spanish Ministry of Science and Innovation from 2009 to 2010, and then as director general of CDTI (the Spanish agency for industrial research) from 2010 to 2012. He is the founder of the international research cente IMDEA Networks, and currently is its director, with a double affiliation as a full arofessor at UC3M.

The use of

Synchronous Ethernet

seems mandatory in

multi-hop scenarios.

Nevertheless, although

high-precision timing

protocols over Ethernet

exist, their accuracy

are in the range of few

hundred nanoseconds,

while CPRI requires at

most tens of

nanoseconds between

REC and RE. 